\title{
Poesia medieval en una carta d'Estanislau Aguiló adreçada a Jaume Massó i Torrents
}

\author{
Helena ROVIRA I CERDÀ \\ helenarovirac@gmal.com
}

\begin{abstract}
RESUM
S'edita una carta d'Estanislau Aguiló dirigida a Jaume Massó i Torrents, datada de 29 de setembre de 1914. Aquest document forma part de l'Arxiu Massó i Torrents de la Biblioteca de Catalunya, i conté informació codicològica sobre tres fulls solts de cançoners catalans medievals, cada un amb una de les següents composicions, algunes fragmentàries: 1) De mala reyl partec erguyl; 2) Se és greu causa per proar; i 3) Rey d'Aragó, ab tota ma desferra. A més, Estanislau Aguiló transcriu el text de les dues primeres peces, i remet a una edició de la tercera obra, publicada per Mateu Obrador i Benassar en 1905.
\end{abstract}

Paraules clau: Jaume Massó i Torrents, epistolari, poesia catalana medieval, edició, proverbis.

[Recibido, julio 2014; aprobado, diciembre 2014]

Medieval poetry in a letter sent by Estanislau Aguiló to Jaume Massó i Torrents

\begin{abstract}
We edit a letter dating from September 29, 1914, written by Estanislau Aguiló and addressed to Jaume Massó i Torrents. This document belongs to Masso i Torrents Archive at the Biblioteca de Catalunya. It contains information on three single sheets from Catalan Medieval songbooks, each containing one of the following compositions, some fragmentary: 1) De mala reyl partec erguyl; 2) Se és greu causa per proar; i 3) Rey d'Aragó, ab tota ma desferra. Furthermore, Estanislau Aguiló transcribed the text of the first two poems and refers to the third one, published by Mateu Obrador i Benassar in 1905.
\end{abstract}

Keywords: Jaume Massó i Torrents, letters, Catalan medieval poetry, edition, proverbs. 
El Repertori de l'antiga literatura catalana de Jaume Massó i Torrents (1932: 35, ítem j) es referia a tres folis solts, provinents de la biblioteca particular d'Estanislau Aguiló. Cada foli transmetia un poema, un d'ells estudiat i reeditat per Jaume Riera i Sans (2009), que l'atribueix a Gilabert de Pròixita ${ }^{1}$. De les altres dues composicions, que continuen essent gairebé desconegudes, n'hi ha a la Biblioteca de Catalunya una transcripció manuscrita de l'any 1914, realitzada per Estanislau Aguiló i dirigida a Jaume Massó i Torrents ${ }^{2}$. Ens trobem davant d'un dels diversos casos en què alguns poemes antics se'ns han conservat en testimonis molt posteriors, deguts en bona mesura al treball de diversos filòlegs $\mathrm{i}$ erudits dels segles XVIII-XX'.

El propòsit d'aquest breu article es donar a conèixer i transcriure els dos fragments poètics i la carta introductòria, que afegeix diverses dades sobre la materialitat d'aquests testimonis. A partir d'ara citarem les dues obres poètiques segons els inicis següents:

\section{1] De mala reyl partec erguyl \\ 2] Se es greu causa per proar}

Es tracta de dues composicions morals o doctrinals, probablement del segle XIV, que no hem pogut identificar. Tot i que podrien ser fragments d'alguna obra occitana o catalana més extensa, potser conservada en altres testimonis que desconeixem, les indagacions que hem fet en aquest aspecte no han donat cap resultat satisfactori ni concret ${ }^{4}$. Els dos poemes tenen una temàtica diferent $\mathrm{i}$ ben determinada. El primer se centra en el vici de l'orgull, i bé podria ser un passatge d'alguna col·lecció sobre vicis i virtuts escrita en vers. El segon és de caire més doctrinal, ja que sobretot parafraseja els aspectes més rellevants del Credo, tot afegint-hi altres matèries d'interès catequètic com són els deu manaments.

Ambdues peces podrien ser acèfales $\mathrm{i}$ àpodes, per bé que aquesta possibilitat sembla menys probable en el primer poema que en el segon. De fet, aquest darrer s'ha de considerar àpode sense cap mena de dubte. La transcripció d'Estanislau Aguiló presenta molts espais en blanc, perquè els folis manuscrits deurien ser il·legibles en aquests mateixos punts. Tot

\footnotetext{
${ }^{1}$ Aquesta composició, també editada per Obrador y Bennassar (1905: [120-121] “Apèndix”), duu la següent rúbrica inicial: "Cobles dictades de un cavaller qui, despagat de son Rey e senyor, enujosament se'n desexia e·1 reptava". Els primers versos diuen, concretament: "Rey d'Aragó, / ab tota ma desferra | Eu prench comiat / e lex la vostra terra, | Rey d'Aragó" (Riera i Sans 2009: 35).

${ }^{2}$ Biblioteca de Catalunya, Fons Massó i Torrents, Correspondència. Carta d'Estanislau Aguiló a Jaume Massó i Torrents, datada de 29 de setembre de 1914. Va acompanyada de dos fulls, cada un d'ells amb la transcripció d'un passatge poètic. Llevat de Massó i Torrents (1913-1914: 227, ítem j; 1932), aquesta font no ha estat inventariada en cap dels principals corpus i repertoris de poesia catalana medieval, com Parramon i Blasco (1993), Mahiques (2009), BITECA (2014) i RIALC (2014).

${ }^{3}$ Aquesta tècnica de transmissió textual seria comparable a la còpia manuscrita d'edicions antigues, recentment estudiada en diversos treballs escrits o aplegats per Martos $(2011 ; 2014)$.

${ }^{4}$ Per les seves característiques, aquests fragments pertanyen al gènere de la literatura didàctica en vers, a la manera del Breviari d'Amor de Matfre Ermengaud, Lo sabi o Libre de Senequa d'autor anònim, la Medicina de peccat i els Proverbis d'ensenyament de Ramon Llull o l'obra proverbial de Cerverí de Girona. Remetem a Ermengaud (19762004), Festa (1906), Pensado (1958), Llull (1938: 3-205; 1928: 373-389) i Guillem de Cervera (1991). Per altra banda, Se es greu causa per proar es pot relacionar amb la tradició de les glosses, traduccions i adaptacions del Credo en llengua vulgar, tema que, tot centrant-se sobretot en la poesia catalana de la segona meitat del segle XV, introdueixen breument Ferrando (2012) i Mahiques (2011).
} 
seguit transcriurem el text de la carta i dels dos poemes. Encara que la carta d'Estanislau Aguiló ha estat escrita al segle XX, la presència de versos medievals i el seu precari estat de conservació ens han fet seguir uns criteris d'edició molt conservadors, intentant moderar la intervenció sobre uns textos tan fragmentaris que difícilment podrien reconstruir-se de manera satisfactòria. De tota manera, hem incorporat algunes propostes de reconstrucció del text tot repetint al marge esquerre alguns versos on figuren entre claudàtors alguns passatges restituïts. Advertim que aquest procediment es fonamenta en el sentit comú i en la probabilitat, sense respondre en cap cas a cap altre criteri metodològic que es pugui establir clarament.

Seguim els mateixos criteris d'edició més comuns utilitzats per a les edicions de textos medievals en català. Resolem en cursiva les abreviatures, marquem entre claudàtors el text afegit i regularitzem segons els usos moderns les alternances entre $i / j$ i $u / v$, la separació de paraules i la combinació de majúscules i minúscules. Hem fet servir les actuals normes ortogràfiques d'accentuació, dièresi, guionets i apòstrofs. Accentuem à, quan es refereix a la tercera persona de singular del present del verb haver; i él, quan és un pronom personal tònic de tercera persona de singular. Hem marcat amb un punt volat les elisions que avui no tenen representació gràfica. Les nombroses llacunes que presenta el text són indicades en punts suspensius. Per altra banda, marquem l'inici del recto i del verso de cada foli a través dels signes $\left|{ }^{\mathrm{r}} \mathrm{i}\right|^{\mathrm{v}}$.

Aquesta transcripció nostra va precedida de la reproducció dels tres fulls que transmeten la carta i els dos poemes del segle XIV.

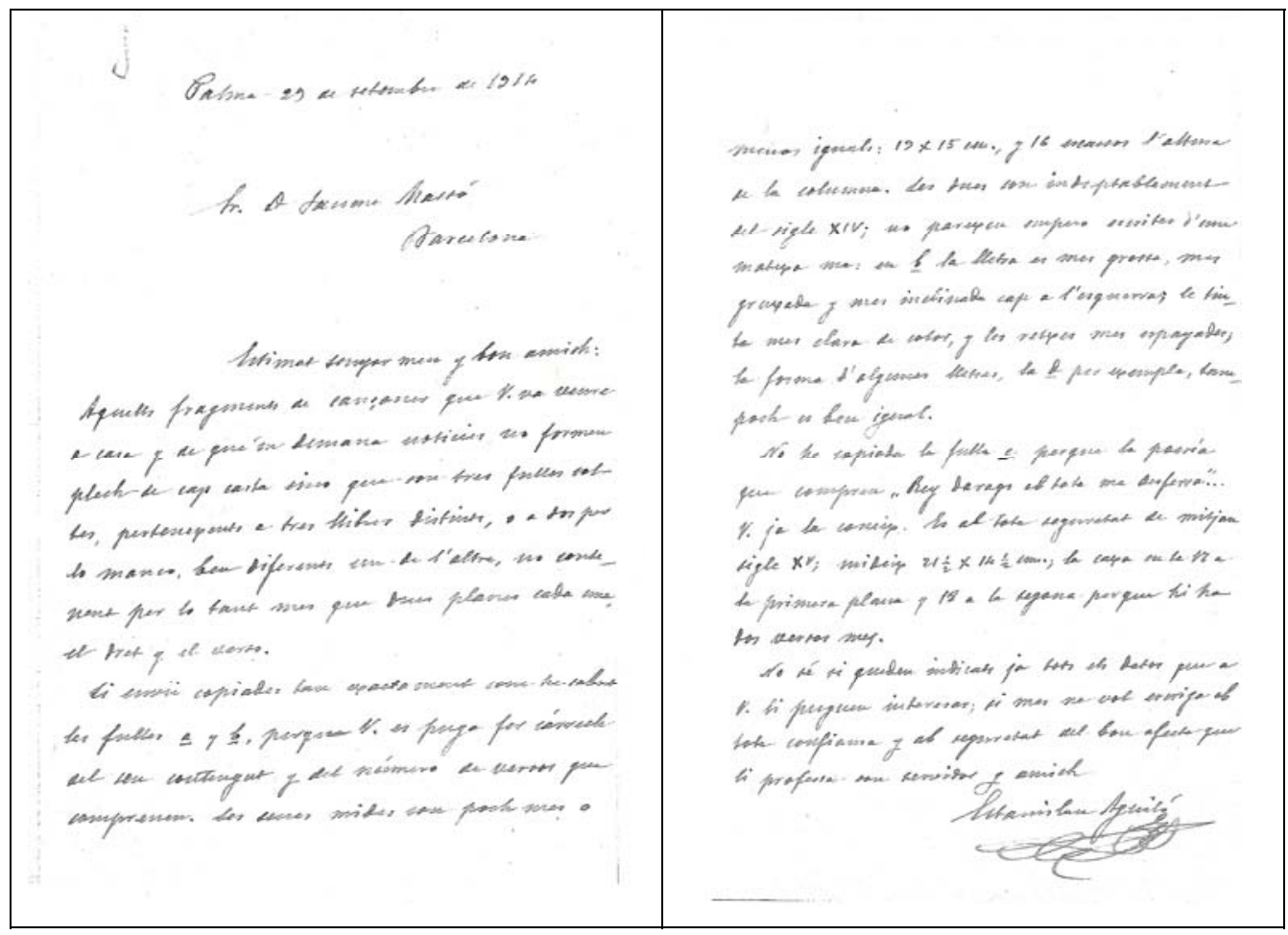




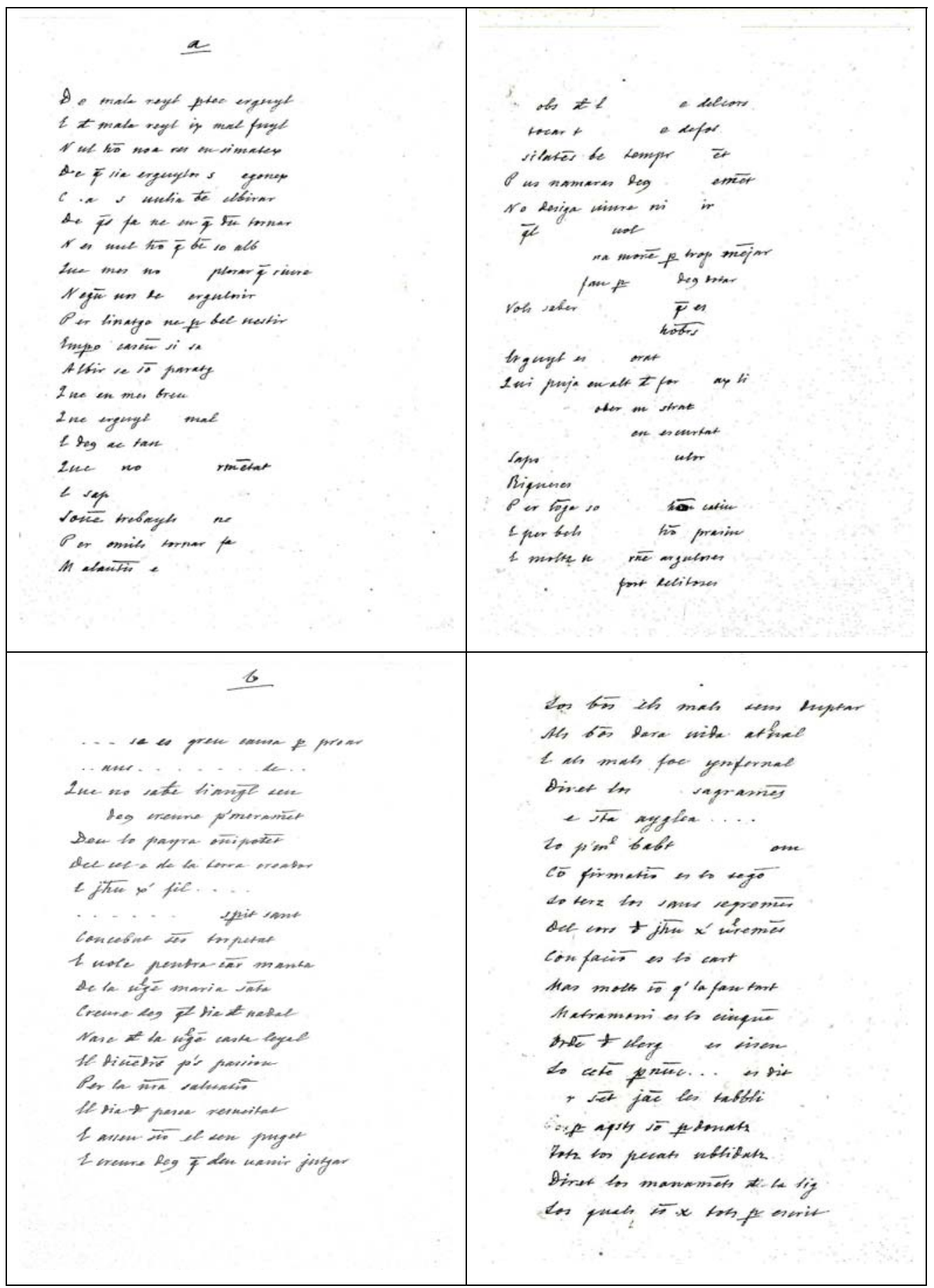


|r Palma, 29 de setembre de 1914

Senyor don Jaume Massó

Barcelona

Estimat senyor meu y bon amich,

aquells fragments de cançoner que vostè va veure a casa y de què· $m$ demanà notícies no formen plech de cap carta sinó que són tres fulles soltes, pertenexents a tres llibres distints, o a dos per lo manco, ben diferents un de l'altre, no contenent per lo tant més que dues planes cada una: el dret y el verso.

Li envïi copiades ben exactament com he sabut les fulles $\boldsymbol{a}$ y $\boldsymbol{b}$, perquè vostè es puga fer càrrech del seu contengut y del número de versos que comprenen. Les seues mides són poch més o $\left.\right|^{\vee}$ menos iguals: $19 \times 15 \mathrm{~cm}$, y 16 escassos l'altura de la columna. Les dues són induptablement del sigle XIV; no parexen, emperò, escrites d'una matexa mà: en $\boldsymbol{b}$ la lletra és més grossa, més gruxada y més inclinada cap a l'esquerra; le tinta més clara de color, y les retxes més espayades. La forma d'algunes lletres, la $d$ per exemple, tampoch és ben igual. No he copiada la fulla $\boldsymbol{c}$, perquè la poesía que comprèn, "Rey d'Aragó, ab tota ma desferra", vostè ja la coneix. És ab tota seguretat de mitjan sigle XV, mideix $21 \frac{1 / 2}{2} \times 14 \frac{1}{2} \mathrm{~cm}$. La caxa en té 17 a la primera plana y 18 a la segona, perquè hi ha dos versos més.

No sé si queden indicats ja tots els datos que a vostè li puguen interesar. Si més ne vol, escriga ab tota confiança y ab seguretat del bon afecte que li professa son servidor y amich,

Estanislau Aguiló $\left.\right|^{\mathrm{r}}$

\begin{tabular}{|c|c|}
\hline & \\
\hline 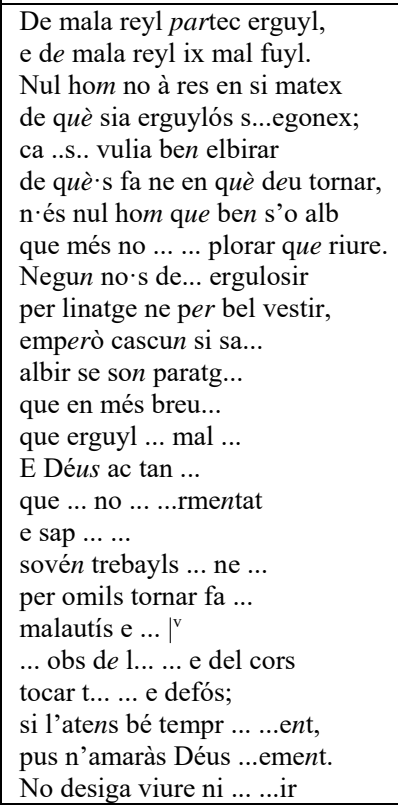 & $\begin{array}{r}\mathrm{ca}[\mathrm{r}] \mathrm{s}[\mathrm{i} \cdot \mathrm{s}] \text { vulia ben elbirar } \\
\text { que més no [am] plorar que riure. } \\
\text { Negun no } \cdot \mathrm{s} \text { de[u] ergulosir } \\
\text { albir se son paratg[e] ... } \\
\text { que ... no ... [tu]rmentat } \\
\\
\text { [per] obs de l'[arma] e del cors } \\
\text { tocar t... [dedins] e defós; } \\
\text { si l'atens bé, tempr[adam]ent, } \\
\text { pus n'amaràs Déus [t]ement. } \\
\text { No desiga viure ni [mor]ir }\end{array}$ \\
\hline
\end{tabular}






e moltz si ...rnen arguloses fort delitoses. $\left.\right|^{\mathrm{r}}$

e moltz si [to]rnen arguloses

\section{b}

... se es greu causa per proar

... ans ... .. de ...

que no saben li àngel seu.

Deus creure primerament

Déu lo Payra omnipotent,

del cel e de la terra creador,

e Jhesuchrist, Fil .......

........ Sperit Sant,

concebut sens torpetat.

E volc pendra carn manta

de la Verge Maria santa.

Creure deus que 1 dia de Nadal

nasc de la Verge casta, leyal,

$\mathrm{e} \cdot 1$ divendres pres passion

per la nostra salvation;

e-1 dia de Pasca, resucitat;

e Assension, Él se'n puget.

E creure deus que deu vanir jutgar $\left.\right|^{\mathrm{v}}$

los bons e-ls mals sens duptar.

Als bons darà vida aternal

e als mals foch ynfernal

Diré't los ...... sagramens

Diré't los [set] sagramens

... e santa ayglea ......

Lo primer babt... ... om;

confirmatión és lo segon;

lo terz, los sans segremens

del cors de Jhesuchrist veremens;

confacion és lo cart,

mas molts són qui la fan tart;

matramoni es lo cinquèn;

orden de clerg....... és sisèn;

orden de clerg[ue] és sisèn;

lo cetèn per nunc... és dit

lo cetèn per $<\mathrm{n}>\mathrm{unc}[\mathrm{ió}]$ és dit

... y sent Jacme l'estabblí.

... per aquests són perdonatz

totz los pecats ublidatz.

Dire't los manaments de la lig, los quals són $\mathrm{X}$, tots per escrit. 


\section{Bibliografía}

BITECA (2014). Beltran, Vicenç / Avenzo, Gemma / Soriano, Lourdes: "Bibliografia de textos antics catalans, valencians i balears", in Philobiblon. Berkeley: Bancroft Library. Data de consulta: 17/07/2014. http://bancroft.berkeley.edu/philobiblon/index_ca.html

CERVERA, Guillem de (1991): Versos proverbials, Joan Coromines (ed.). Barcelona: Curial.

ERMENGAUD, Matfre (1976-2004): Le Breviari d'amor, Peter E. Rickets (ed.), 5 vol. Leiden: Brill / Turnhout: Brepols.

FERRANDO, Antoni (2012): "Noves aportacions a Narcís Vinyoles. Edició de 'Lo Credo in Deum aplicat per sos articles a la Mare de Déu d'Esperança y los goigs",, in Estudios sobre el Cancionero General (Valencia, 1511), poesía, manuscrito e imprenta, Marta Haro Cortés, Rafael Beltrán Llavador, José Luis Canet Vallés y Héctor H. Gassó (coords.), vol. 2, pp. 479-506. València: Universitat de València.

Festa, Giovanni Battista (1906): "Le 'Savi ou Libre de Senequa' d'après les deux manuscrits de Rome et de Paris". Annales du Midi 18/69: 297-325.

Llull, Ramon (1928): Proverbis de Ramon. Mil proverbis. Proverbis d'ensenyament. Transcripció directa amb facsímil, introducció $i$ variants dels més vells manuscrits, Salvador Galmés (ed.). Palma de Mallorca: Diputació Provincial de Balears / Institut d'Estudis Catalans. [Obres de Ramon Llull, vol. XIV].

Llull, Ramon (1938): Rims: Medicina de peccat, Aplicació de l'Art General, Del consili, Cantilena, Salvador Galmés (ed.). Palma de Mallorca: Diputació Provincial de Balears / Institut d'Estudis Catalans de Barcelona. [Obres de Ramon Llull, vol. XX, tom II]. 